\section{Avaliação do declínio cognitivo e sua relação com as características socioeconômicas dos idosos em Viçosa-MG}

\section{Evaluation of cognitive decline and its relationship with socioeconomic characteristics of the elderly in Viçosa, Minas Gerais}

Juliana Costa Machado'

Rita de Cássia Lanes Ribeiro²

Paulo Fernando da Glória Leal ${ }^{3}$

Rosângela Minardi Mitre Cotta ${ }^{4}$

Departamento de Nutrição e Saúde, Universidade Federal de Viçosa, MG, Brasil

Endereço para correspondência: Profa. Dra. Rita de Cassia Lanes Ribeiro. Universidade Federal de Viçosa MG Brasil - Departamento de Nutrição e Saúde DNS. Avenida P.H, Rolfs s/n Campus Universitário - CEP 36.570 .000 - Viçosa MG Brasil. E-Mail: rribeiro@ufv.br.

\section{Resumo}

Introdução: Este estudo teve como objetivo avaliar o declínio cognitivo de idosos (indivíduos com 60 anos ou mais), freqüentadores do Programa Municipal da Terceira Idade (PMTI), em Viçosa, MG, e sua relação com as características socioeconômicas. Métodos: Realizou-se um estudo analítico transversal com 74 idosos. Foram incluídos no estudo os idosos alfabetizados que preencheram os seguintes critérios: ausência de perda visual ou auditiva, eventualmente corrigidas, ausência de alterações motoras e paralisia nos membros superiores ou a falta deles, ausência de problemas neurológicos ou psiquiátricos que prejudicassem o desempenho no Mini-Exame do estado mental (MEEM). Os instrumentos de coleta de dados adotados foram um questionário, elaborado pela equipe de pesquisadores para a avaliação das características socioeconômicas, e o MEEM. Resultados: Dentre os idosos avaliados, encontrou-se uma freqüência de declínio cognitivo de $36,5 \%$. A média de pontuação no MEEM foi de 19,48 pontos em um total de 30 pontos e a média da idade dos idosos foi de 68,5 anos. Discussão: Os resultados encontrados mostram que o menor nível de escolaridade tem influência negativa no estado mental dos idosos. Conclusão: Conclui-se que é alto o número de idosos identificados com declínio cognitivo. Para se concluir um diagnóstico de demência em idosos, cujas respectivas pontuações foram abaixo do determinado, é necessária uma avaliação neuropsicológica mais apurada.

Palavras-chave: Idoso. Avaliação. Declínio cognitivo. Mini-Exame do Estado Mental. Demência. 


\section{Abstract}

Introduction: This study assesses cognitive decline in individuals 60 years of age and over, participants of a City Third Age Program (CPTA) in Viçosa, MG, and its relation with social and economic characteristics. Methods: A cross-sectional analytical study was carried out with 74 elderly individuals. The study included literate individuals who filled out the following criteria: absence of visual or auditory loss, with some inclusions of corrected cases; absence of motor alterations, upper member paralysis or lack of members; absence of neurological or psychiatric problems affecting performance in the MMSE (MiniMental State Examination). Data collecting tools were a questionnaire prepared by the research team, and the MMSE. Results: The frequency of cognitive decline was $36.5 \%$. The average MMSE score was 19.48 points of a best total of 30 , and the average age of the elderly was 68.5 years. Discussion: The results showed that a lower level of schooling has a negative influence on the mental state of the elderly. Conclusion: A high number of elderly individuals with cognitive decline were identified, which is an unsettling factor. To conclude, the diagnosis of dementia in the elderly whose scores indicated cognitive decline needs more refined neurological and psychiatric evaluation.

Keywords: Aged. Cognitive decline. MiniMental State Examination. Dementia.

\section{Introdução}

Os temas relacionados ao envelhecimento vêm sendo cada vez mais estudados em decorrência do aumento da população idosa no mundo. Nos países desenvolvidos, esse aumento ocorreu lentamente, permitindo um planejamento de políticas públicas para que essas mudanças demográficas não interferissem negativamente na qualidade de vida da população. No Brasil, assim como em outros países em desenvolvimento, esse fenômeno é recente e vem ocorrendo de forma rápida, apresentando para a sociedade o desafio de se adaptar a essa nova realidade ${ }^{1}$.

O Plano de Ação Internacional sobre o Envelhecimento das Nações Unidas ${ }^{2}$ recomenda que, para países em desenvolvimento, deva-se considerar como idoso a pessoa com 60 anos e mais. No Brasil, a Política Nacional do Idoso também estipulou 60 anos como o patamar que caracteriza o grupo de idosos ${ }^{3}$.

Segundo a Organização Mundial de Saúde (OMS) estima-se que entre 1950 e 2025 o número de idosos no Brasil deverá ter aumentado em quinze vezes, enquanto as outras faixas etárias cinco vezes. Assim, o Brasil será o sexto país quanto ao contingente de idosos em 2025, devendo ter cerca de 32 milhões de pessoas com mais de 60 anos $^{4}$.

Atualmente, no Brasil, os idosos representam cerca de $10 \%$ da população total. O censo de 2000 informou que 15,5 milhões de brasileiros têm mais de 60 anos, sendo estes na maioria mulheres, viúvas, com baixa escolaridade e baixa renda. $\mathrm{O}$ processo de envelhecimento pode ser acompanhado pelo declínio das capacidades tanto físicas como cognitivas dos idosos, de acordo com suas características de vida. À medida que o número de idosos aumenta na população, é necessário um conhecimento maior de suas necessidades e o acompanhamento de suas mudanças.

Necessita-se de informação atualizada deste segmento, no campo da demografia, das mudanças no entorno familiar, das 
questões sociais, da situação econômica e de saúde ${ }^{1,5,6}$.

Dentre as doenças de caráter crônico, a demência é uma das mais importantes causas de morbimortalidade e, hoje é o problema de saúde mental que mais cresce em importância e número, com estimativas apontando, com o envelhecimento, um aumento na incidência anual, sendo $0,6 \%$, na faixa dos $65-69$ anos e $8,4 \%$ naqueles com mais de 85 anos, e sua prevalência aumentando exponencialmente com a idade, passando de $5 \%$ entre aqueles com mais de 60 anos, para $20 \%$ naqueles com idade superior a $80 \operatorname{anos}^{7,8}$.

De acordo com Lopes e Botino ${ }^{9}$, a prevalência de demência variou entre $2,2 \%$ na África, 5,5\% na Ásia, 6,4\% na América do Norte, $7,1 \%$ na América do Sul e 9,4\% na Europa. No Brasil, descreveu-se uma taxa de prevalência intermediária $(7,1 \%)$ entre os resultados dos estudos internacionais.

As síndromes demenciais são caracterizadas pela presença de declínio cognitivo progressivo, cujas características principais são: declínio da memória adquirido, declínio intelectual ou de outras funções cognitivas, mudanças no comportamento ou na personalidade, além do prejuízo no desenvolvimento psicossocial. O grau de incapacidade aumenta com o avanço do declínio cognitivo. O diagnóstico de demência baseia-se principalmente na presença de declínio da memória e de outras funções corticais superiores, tais como linguagem, praxia, capacidade de reconhecer e identificar objetos, abstração, organização, capacidade de planejamento e seqüenciamento $^{7,10,11}$.

O comprometimento das funções cognitivas é caracterizado, na demência, pela deterioração progressiva das habilidades intelectuais, como, por exemplo, a perda da capacidade de julgamento, da memória, do raciocínio abstrato e das habilidades visuo-espaciais, que afetam a capacidade funcional do indivíduo no seu dia a dia, implicando em perda de independência e autonomia, a qual varia de acordo com o grau de gravidade, com conseqüente perda da qualidade de vida do idoso ${ }^{11}$. O impacto da demência e das desordens a ela relacionadas, sobre a família do idoso e a sociedade não deve ser subestimado. Vários são os fatores de risco a ela associados, tais como: a natureza marcadamente dependente da idade que caracteriza a deficiência cognitiva, a predominância em mulheres, a acentuada importância dos fatores socioeconômicos e a associação com a incapacidade de desempenhar as atividades cotidianas ${ }^{12,13}$.

Estudos sobre capacidades cognitivas da população idosa objetivam avaliar em um primeiro estágio a prevalência de declínio cognitivo e, para tal, durante os últimos anos foram desenvolvidos instrumentos com o objetivo de auxiliar na investigação de possíveis déficits cognitivos em indivíduos de risco, como é o caso dos idosos ${ }^{7}$.

O Mini-Exame do Estado Mental (MEEM) é uma escala de avaliação cognitiva que auxilia na investigação e na monitoração da evolução de possíveis déficits cognitivos em pessoas com risco de demência. Desde que foi proposto por Folstein et al. ${ }^{14}$, vem sendo amplamente utilizado no mundo. No Brasil, o MEEM foi inicialmente proposto por Bertolucci et al. ${ }^{15}$, que observaram que o escore total do MEEM dependia do nível educacional. Tombaugh e McIntyre ${ }^{16}$ observaram que a escala tem boa consistência interna e confiabilidade teste-reteste. Também demonstraram que o ponto de corte 23/24 tem boa a excelente sensibilidade e especificidade para o diagnóstico de demência.

A demência pode ser definida como um declínio global e progressivo da memória, do intelecto, da crítica e da personalidade, sendo considerada uma síndrome de perda adquirida das funções cognitivas, de alterações no comportamento e perda de funções sociais. Muitas doenças têm a demência como uma de suas manifestações, e existem doenças que são exclusivamente a demência. A Doença de Alzheimer é a causa mais freqüente de demência ${ }^{17}$. Es- 
tudos epidemiológicos mostram que idosos com declínio cognitivo apresentam maior risco de desenvolver Doença de Alzheimer $^{8,18}$. Essas enfermidades têm impacto sobre a família e sobre os cuidadores dos idosos, representando um problema significativo de saúde pública, por sua longa extensão e complexidade de manifestações funcionais, emocionais e conseqüências sociais. Os familiares ou a pessoa que zela pelo idoso nem sempre conseguem identificá-las, pois são complexas e progressivas, e muitas vezes consideradas "normais" na velhice. A falta do diagnóstico pode não só aumentar os danos à saúde mental e ao bem-estar do idoso, como também trazer riscos de acidentes com medicamentos, no ambiente doméstico ou fora dele. Nesse sentido, faz-se necessário que os profissionais da saúde conheçam e saibam diagnosticar os sintomas dessas enfermidades, bem como se comprometam e sejam capazes de desenvolver ações efetivas que promovam a melhoria na qualidade de vida dos idosos no âmbito individual e coletivo.

O objetivo do presente estudo foi avaliar o declínio cognitivo e sua relação com as características socioeconômicas de idosos.

\section{Materiais e Métodos}

\section{O Desenho do Estudo}

Trata-se de um estudo epidemiológico em que se utilizou o método analítico do tipo transversal para investigar a presença ou não de declínio cognitivo, e sua relação com as características socioeconômicas dos idosos, participantes do Programa Municipal da Terceira Idade (PMTI) do município de Viçosa, MG. Foram utilizados dois instrumentos para a coleta de dados. Para avaliar as características socioeconômicas, foi criado pela equipe de pesquisadores um questionário específico para este estudo, tendo como referência trabalhos presentes na literatura ${ }^{7,19-22}$. Para identificar a presença de declínio cogni- tivo, foi utilizado o teste do Mini-Exame do Estado Mental (MEEM), versão em português validada por Bertolucci et al., em $1994^{15}$.

\section{Métodos}

A população deste estudo foi constituída por idosos selecionados por amostragem aleatória simples a partir do cadastro do PMTI. Este programa foi instituído em 1997, sendo resultado de uma parceria entre o Núcleo de Saúde Pública (NUSP) do Departamento de Nutrição e Saúde (DNS) da Universidade Federal de Viçosa (UFV) com a Prefeitura Municipal de Viçosa (PMV), e cujo objetivo principal é promover, por meio de um processo educativo-preventivo, a melhoria da qualidade de vida dos idosos. O PMTI conta com 1.025 idosos que participam de atividades físicas e de recreação, tendo acesso a atendimento médico, odontológico e nutricional.

Dadas as características do estudo, incluiu-se no sorteio os idosos que preencheram os seguintes critérios: ausência de perda visual ou auditiva, eventualmente corrigidas, ausência de alterações motoras e paralisia nos membros superiores ou falta deles, ausência de problemas neurológicos ou psiquiátricos que prejudicassem o desempenho no MEEM. No início do estudo foi aplicado um pré-teste em que foram identificadas dificuldades nas respostas do MEEM por parte dos idosos analfabetos. Portanto, incluíram-se neste estudo os idosos que eram pelo menos alfabetizados?.

A amostra foi calculada considerando uma prevalência de declínio cognitivo de no mínimo $5 \%$ da população de idosos ${ }^{7}$. Para o cálculo do tamanho da amostra, utilizou-se a equação proposta por Lwanga e Lemeshow $^{23}$, com um poder de estudo de $90 \%$ e um erro de $5 \%$; deste modo, foram avaliados 74 idosos.

O dados foram obtidos na sede do PMTI, por meio de entrevistas individuais realizadas por um único entrevistador treinado. Os idosos que aceitaram participar 
do estudo assinaram o Termo de Consentimento Livre Esclarecido e, em um primeiro momento, responderam o questionário de avaliação das características socioeconômicas cujas variáveis avaliadas foram:

- Socioeconômicas: sexo, cor, faixa etária, grau de instrução, estado civil, número de pessoas que residem no domicílio, número de dependentes e de pessoas que trabalham na família, condições de moradia, se exerceu atividade remunerada, se recebe pensão ou aposentadoria e renda domiciliar mensal;

- Fatores relacionados à sociabilidade, lazer e autopercepção dos seus relacionamentos: tipos de atividades de lazer de preferência dos idosos, se eles se sentem satisfeitos com essas atividades, quais os fatores que os deixam insatisfeitos com o lazer e o que os impede de fazer outras atividades no cotidiano, satisfação com o relacionamento familiar.

Em um segundo momento aplicou-se o teste do MEEM, que é composto por diversas questões agrupadas em 7 categorias, sendo que cada uma delas objetiva avaliar déficits das funções cognitivas específicas: orientação para o tempo (5 pontos), orientação para o local (5 pontos), registro de três palavras (3 pontos), atenção e cálculo (5 pontos), lembrança de três palavras (3 pontos), linguagem (8 pontos) e capacidade construtiva visual (1 ponto). $\mathrm{O}$ escore do MEEM pode variar de um mínimo de 0 até o total máximo de 30 pontos.

Neste estudo foram considerados portadores de declínio cognitivo os idosos que apresentaram escore abaixo de 23 no MEEM, pois, segundo Almeida ${ }^{7}$, para idosos com algum tipo de instrução o ponto de corte deve ser de 23/24, pois esse escore está associado à sensibilidade de $78 \% \mathrm{e}$ especificidade de $75 \%$. Para a interpretação dos resultados, considerou-se a existência de associação entre um determinado fator de estudo (variáveis socioeconômicas) e a presença de declínio cognitivo.
Os dados foram digitados no programa Epi-Info, versão 6.02. Foi realizada uma análise descritiva e uma análise bivariada, bem como estimados os valores de odds ratio com intervalos de confiança de $95 \%$.

O presente estudo foi aprovado pelo Comitê de Ética em Pesquisa com Seres Humanos, da Universidade Federal de Viçosa.

\section{Resultados}

Dos 74 entrevistados, 27 apresentaram declínio cognitivo (36,5\%). A média de pontuação no MEEM foi de 19,48 pontos (menor pontuação = 13; maior pontuação =28) e a média da idade foi de 68,5 anos (com variação de 60 a 85 anos).

Com relação à faixa etária, constatouse uma predominância de idosos com idade entre 60 e 69 anos (65\%), seguidos dos que têm entre 70 e 79 anos (32\%), e mais de 80 anos (3\%). Quando se relaciona a faixa etária com o declínio cognitivo, verifica-se que a maioria dos idosos com declínio cognitivo tem idade entre 60-69 anos (24,32\%), seguidos dos que têm entre 70 79 anos (10,81\%) e daqueles com mais de 80 anos $(1,75 \%)$.

Observa-se uma maior proporção de idosos do sexo feminino (74\%), de cor parda $(67 \%)$ e de baixa escolaridade $(91 \%$ têm menos de 4 anos de estudo). Quanto à situação conjugal, constatou-se que a maioria dos idosos é casada (60\%). Com relação ao arranjo familiar no domicílio: $28 \%$ moram com o cônjuge; $46 \%$ moram com o cônjuge ou não, e com filhos; $12 \%$ moram com o cônjuge ou não, filhos e netos; e $14 \%$ moram sozinhos. Destaca-se que a maioria dos idosos $(86 \%)$ se diz satisfeita com o seu relacionamento familiar (Tabela 1).

A maioria dos idosos possui dependentes $(66 \%)$, porém vive em famílias em que uma ou mais pessoas estão trabalhando (66\%). Um dado importante é a predominância de idosos residentes em casa própria (92\%).

Os dados relativos à situação econômica revelam que $76 \%$ dos idosos exerceram 
Tabela 1 - Distribuição dos idosos com declínio cognitivo e sem declínio cognitivo segundo características sociais e econômicas. Município de Viçosa, Minas Gerais (2005).

Table 1 - Distribution of the elderly with cognitive decline and without cognitive decline according to social and economical characteristics. Municipal district of Viçosa, Minas Gerais (2005).

\begin{tabular}{|c|c|c|c|c|c|c|c|}
\hline \multirow[t]{2}{*}{ Variáveis } & \multirow[t]{2}{*}{ Categorias } & \multicolumn{2}{|c|}{ Total } & \multicolumn{2}{|c|}{$\begin{array}{l}\text { Com Declínio } \\
\text { Cognitivo }\end{array}$} & \multicolumn{2}{|c|}{$\begin{array}{c}\text { Sem Declínio } \\
\text { Cognitivo }\end{array}$} \\
\hline & & $\mathrm{N}$ & $\%$ & $\mathrm{~N}$ & $\%$ & $\mathrm{~N}$ & $\%$ \\
\hline \multirow{3}{*}{ Faixa Etária } & $60-69$ anos & 48 & 65 & 18 & 24,32 & 30 & 40,5 \\
\hline & $70-79$ anos & 24 & 32 & 8 & 10,81 & 16 & 21,6 \\
\hline & 80 ou mais & 2 & 3 & 1 & 1,75 & 1 & 1,35 \\
\hline \multirow[t]{2}{*}{ Sexo } & Masculino & 19 & 26 & 3 & 4,05 & 16 & 21,6 \\
\hline & Feminino & 55 & 74 & 24 & 32,4 & 31 & 41,9 \\
\hline \multirow[t]{3}{*}{ Cor } & Branca & 10 & 14 & 5 & 6,75 & 5 & 6,75 \\
\hline & Preta & 14 & 19 & 6 & 8,1 & 8 & 10,8 \\
\hline & Parda & 50 & 67 & 16 & 21,62 & 34 & 45,94 \\
\hline \multirow{3}{*}{$\begin{array}{l}\text { Escolaridade Completa } \\
\text { (em Anos) }\end{array}$} & 1 ano ou menos & 36 & 50 & 19 & 25,67 & 17 & 22,9 \\
\hline & $1-4$ anos & 31 & 41 & 7 & 9,45 & 24 & 32,4 \\
\hline & 4 ou mais & 7 & 9 & 1 & 1,75 & 6 & 8,1 \\
\hline \multirow[t]{2}{*}{ Estado Civil } & Solteiro/Viúvo/Separado & 30 & 40 & 13 & 17,56 & 17 & 22,97 \\
\hline & Casado & 44 & 60 & 14 & 18,9 & 30 & 40,54 \\
\hline Arranjo Familiar & Só & 10 & 14 & 3 & 4,05 & 7 & 9,45 \\
\hline \multirow[t]{3}{*}{ no Domicilio } & Cônjuge & 21 & 28 & 7 & 9,45 & 14 & 18,9 \\
\hline & $\begin{array}{l}\text { Com Cônjuge ou } \\
\text { não e filhos }\end{array}$ & 34 & 46 & 14 & 18,9 & 20 & 27 \\
\hline & $\begin{array}{l}\text { Com Cônjuge ou não, } \\
\text { filhos, netos e outros }\end{array}$ & 9 & 12 & 3 & 4,05 & 6 & 8,1 \\
\hline Satisfação com & Sim & 64 & 86 & 23 & 31,08 & 41 & 55,4 \\
\hline \multirow[t]{2}{*}{ Relacionamento Familiar } & Não & 6 & 8 & 4 & 5,4 & 2 & 2,7 \\
\hline & $\begin{array}{l}\text { Não sabem ou } \\
\text { responderam }\end{array}$ & 4 & 6 & 0 & 0 & 4 & 5,4 \\
\hline \multirow[t]{2}{*}{ Número de Dependentes } & Nenhum & 25 & 34 & 11 & 14,86 & 14 & 18,91 \\
\hline & 1 ou mais & 49 & 66 & 16 & 21,62 & 33 & 44,59 \\
\hline Número de Pessoas da & Nenhum & 25 & 34 & 17 & 22,97 & 20 & 27,02 \\
\hline Família Que Trabalham & 1 ou mais & 49 & 66 & 10 & 13,5 & 27 & 36,48 \\
\hline \multirow[t]{2}{*}{ A Moradia é } & Própria & 68 & 92 & 25 & 33,78 & 43 & 58,1 \\
\hline & Alugada / ou de Parentes & 6 & 8 & 2 & 2,7 & 4 & 5,4 \\
\hline Exerceu Atividade & Sim & 56 & 76 & 19 & 25,67 & 37 & 50 \\
\hline Remunerada & Não & 18 & 24 & 8 & 10,81 & 10 & 13,5 \\
\hline \multirow[t]{2}{*}{ Recebendo Aposentadoria } & Sim & 47 & 64 & 15 & 20,27 & 32 & 43,24 \\
\hline & Não & 27 & 36 & 12 & 16,2 & 15 & 20,27 \\
\hline \multirow[t]{2}{*}{ Recebendo Pensão } & Sim & 22 & 30 & 10 & 13,51 & 12 & 16,2 \\
\hline & Não & 52 & 70 & 17 & 22,97 & 35 & 47,29 \\
\hline \multirow[t]{4}{*}{ Renda Domiciliar Mensal } & < 1 Salário Mínimo & 2 & 3 & 0 & 0 & 2 & 2,7 \\
\hline & 1 - 3 Salários Mínimos & 66 & 89 & 26 & 35,1 & 40 & 54,05 \\
\hline & 4 - 5 Salários Mínimos & 4 & 5 & 1 & 1,35 & 3 & 4,05 \\
\hline & 5 ou Mais & 2 & 3 & 0 & 0 & 2 & 2,7 \\
\hline
\end{tabular}


atividade remunerada formal durante a vida, sendo que $64 \%$ declararam receber aposentadoria e $30 \%$ declararam receber pensão.

A média da renda domiciliar mensal encontrada foi de 690 reais (variando de 559,25 reais para os idosos com declínio cognitivo, e 765,31 para os idosos sem declínio), sendo que $89 \%$ dos idosos disseram receber entre 1 a 3 salários mínimos, e 5\% entre 4-5 salários mínimos (o valor de 1 salário mínimo, na ocasião do estudo, era de 300,00 reais).

No que se refere à sociabilidade e ao lazer, pode-se verificar que os tipos de atividades de preferência dos idosos em seus momentos de lazer foram: ir à igreja (81\%), visitar parentes (76\%) e assistir TV (62\%). Em relação à satisfação com as atividades de lazer, enfatiza-se que $85 \%$ dos idosos consideram-se satisfeitos com o seu lazer no cotidiano. Quando solicitados a avaliar qual o principal motivo de insatisfação com as atividades desempenhadas no seu tempo livre, tanto os idosos que se disseram satisfeitos com seu lazer quanto os que se disseram insatisfeitos, relataram os problemas com o custo financeiro dessas atividades como o principal motivo de insatisfação (46\%), seguidos dos problemas de saúde (14\%) e da falta de motivação (8\%) (Tabela 2).

$\mathrm{Na}$ Tabela 3 estão apresentados os resultados da análise dos fatores socioeconômicos relacionados ao declínio cognitivo. Na avaliação cognitiva dos idosos, realizada por meio do MEEM, chama a atenção a associação entre a escolaridade e a presença de declínio cognitivo. A razão de chances (odds ratio) mostra que os idosos com um ano ou menos de estudo têm 3,83 vezes mais chances de ter declínio cognitivo do que os que têm de 1-4 anos de estudo. A razão de chances entre as demais variáveis socioeconômicas e o declínio cognitivo não se mostrou estatisticamente significante.

Quando foi analisada a associação entre os fatores relacionados à sociabilidade e ao lazer no cotidiano e o declínio cogniti- vo não se observou associação estatisticamente significante (Tabela 4).

\section{Discussão}

Os resultados mostram um elevado número de idosos com declínio cognitivo quando comparados a alguns estudos ${ }^{9,21,22}$. Uma possível explicação para este resultado seria a escolha do ponto de corte tradicional no MEEM (23 pontos) utilizado, que se mostrou elevado para idosos com baixa escolaridade. Porém, como era desejável neste estudo, aumentou a sensibilidade do teste para a detecção de um número maior de casos verdadeiros, ao mesmo tempo em que diminuiu a possibilidade de idosos com um grau "leve" de declínio cognitivo não serem diagnosticados.

Segundo Almeida ${ }^{7}$, a utilização do MEEM como instrumento de "screening" para demência sugere ser mais desejável empregar pontos de corte mais elevados para aumentar a sensibilidade do teste, detectando-se com isso o maior número possível de casos verdadeiros.

O declínio cognitivo dificulta a realização das atividades da vida diária e as relações sociais e familiares, prejudicando gradativamente a autonomia do idoso. $\mathrm{Na}$ velhice, a manutenção da qualidade de vida está intimamente ligada à capacidade do idoso de desempenhar as funções necessárias à manutenção da sua vida diária e prática, de modo a torná-lo independente dentro do seu contexto socioeconômico e cultural ${ }^{19}$. O diagnóstico precoce de declínio cognitivo possibilita intervenção terapêutica, diminui os níveis de estresse para os familiares, reduz o risco de acidentes, prolonga a autonomia e, em alguns casos, retarda o início do processo demencial $^{18}$. Nesse sentido, destaca-se a importância de estudos sobre essa doença para orientação e implementação de políticas de saúde pública.

O MEEM é um instrumento de rastreio do declínio cognitivo, sugerindo-se que sujeitos com escores inferiores ao ponto 
Tabela 2 - Distribuição dos idosos com declínio cognitivo e sem declínio cognitivo segundo fatores relacionados à sociabilidade e lazer. Município de Viçosa (2005).

Table 2 - Distribution of the elderly with cognitive decline and without cognitive decline according to factors related to sociability and leisure. Municipal district of Viçosa, Minas Gerais (2005).

\begin{tabular}{|c|c|c|c|c|c|c|c|}
\hline \multirow[t]{2}{*}{ Variáveis } & \multirow[t]{2}{*}{ Categorias } & \multicolumn{2}{|c|}{ Total } & \multicolumn{2}{|c|}{$\begin{array}{c}\text { Com Declínio } \\
\text { Cognitivo }\end{array}$} & \multicolumn{2}{|c|}{$\begin{array}{c}\text { Sem Declínio } \\
\text { Cognitivo }\end{array}$} \\
\hline & & $\mathrm{N}$ & $\%$ & $\mathrm{~N}$ & $\%$ & $\mathrm{~N}$ & $\%$ \\
\hline \multirow[t]{2}{*}{ Rádio } & Sim & 21 & 28 & 9 & 12.16 & 12 & 16.21 \\
\hline & Não & 53 & 73 & 18 & 24.32 & 35 & 47.29 \\
\hline \multirow[t]{2}{*}{ TV } & Sim & 46 & 62 & 19 & 25,67 & 27 & 36,48 \\
\hline & Não & 28 & 38 & 8 & 10,81 & 20 & 27 \\
\hline \multirow[t]{2}{*}{ Ler Jornal, Revista, Livro } & Sim & 29 & 39 & 7 & 9.45 & 22 & 29.7 \\
\hline & Não & 45 & 61 & 20 & 27 & 25 & 33,78 \\
\hline \multirow[t]{2}{*}{ Visitar Parentes } & Sim & 56 & 76 & 21 & 28.37 & 35 & 47.29 \\
\hline & Não & 18 & 24 & 6 & 8,1 & 12 & 16,21 \\
\hline \multirow[t]{2}{*}{ Andar Pelo Bairro } & Sim & 20 & 27 & 7 & 9.45 & 13 & 17.56 \\
\hline & Não & 54 & 73 & 20 & 27 & 34 & 45.94 \\
\hline \multirow[t]{2}{*}{ Ir à Igreja } & Sim & 60 & 81 & 22 & 29.72 & 38 & 51.35 \\
\hline & Não & 14 & 19 & 5 & 6.75 & 9 & 12.16 \\
\hline \multirow[t]{2}{*}{ Ir à Jogos } & Sim & 15 & 20 & 4 & 5.4 & 11 & 14.86 \\
\hline & Não & 59 & 80 & 23 & 31.08 & 36 & 48.64 \\
\hline \multirow{2}{*}{ Fazer Compras } & Sim & 3 & 4 & 1 & 1.35 & 2 & 2.7 \\
\hline & Não & 71 & 96 & 26 & 35.13 & 45 & 60.81 \\
\hline \multirow[t]{2}{*}{ Passeios Longos } & Sim & 16 & 22 & 6 & 8.1 & 10 & 13.5 \\
\hline & Não & 58 & 78 & 21 & 28.37 & 37 & 50 \\
\hline \multirow[t]{2}{*}{ Outras Atividades } & Sim & 42 & 57 & 12 & 16.21 & 30 & 40.54 \\
\hline & Não & 32 & 43 & 15 & 20.27 & 17 & 22.97 \\
\hline Satisfeito com as & Sim & 63 & 85 & 22 & 29.72 & 41 & 55.4 \\
\hline \multirow[t]{3}{*}{ Atividades Que faz } & Não & 10 & 13 & 5 & 6.75 & 5 & 6.75 \\
\hline & Não Sabem ou & 1 & 2 & 0 & 0 & 1 & 1.35 \\
\hline & Não Responderam & & & & & & \\
\hline Motivo da Insatisfação & Problema de Custo & 34 & 46 & 16 & 21.62 & 18 & 24.32 \\
\hline Com as Atividades & Problema de Saúde & 10 & 14 & 5 & 6.75 & 5 & 6.75 \\
\hline Desempenhados no & Falta de Motivação & 6 & 8 & 0 & 0 & 6 & 8.1 \\
\hline \multirow[t]{4}{*}{ Tempo Livre } & Problema com & 4 & 5 & 2 & 2.7 & 2 & 2.7 \\
\hline & Transporte & & & & & & \\
\hline & Outras Razões & 6 & 8 & 2 & 2.7 & 4 & 5.4 \\
\hline & Não Sabem & 14 & 19 & 2 & 2.7 & 12 & 16.21 \\
\hline
\end{tabular}


Tabela 3 - Razão de chances entre as variáveis sociais e econômicas com a presença ou não de declínio cognitivo. Município de Viçosa, MG (2005).

Table 3 - Odds ratio among the social and economic variables with the presence or not of cognitive decline. Municipal district of Viçosa, Minas Gerais (2005).

\begin{tabular}{|c|c|c|}
\hline Variáveis & Categorias & OR (IC 95\%) \\
\hline \multirow[t]{3}{*}{ Faixa Etária } & *60 - 69 anos & 1,0 \\
\hline & $70-79$ anos & $1,20(0,38-3,82)$ \\
\hline & 80 ou mais & $0,60(0,02-23,69)$ \\
\hline \multirow[t]{2}{*}{ Sexo } & Masculino & $0,24(0,05-1,04)$ \\
\hline & Feminino & \\
\hline \multirow[t]{3}{*}{ Cor } & *Branca & 1,0 \\
\hline & Preta & $1,33(0,19-9,33)$ \\
\hline & Parda & $2,13(0,45-10,23)$ \\
\hline \multirow[t]{3}{*}{ Escolaridade Completa (em Anos) } & *1 ano ou menos & 1,0 \\
\hline & $1-4$ anos & $3,83(1,18-12,88)$ \\
\hline & 4 ou mais & $6,71(0,66-163,53)$ \\
\hline \multirow[t]{2}{*}{ Estado Civil } & Solteiro/Viúvo/Separado & $0,61(0,21-1,78)$ \\
\hline & Casado & \\
\hline \multirow[t]{4}{*}{ Arranjo Familiar no Domicílio } & *Só & 1,0 \\
\hline & Cônjuge & $0,86(0,12-5,62)$ \\
\hline & Com Cônjuge ou Não e Filhos & $0,61(0,10-3,36)$ \\
\hline & Com Cônjuge ou Não, Filhos, Netos & $1,71(0,08-60,14)$ \\
\hline \multirow[t]{2}{*}{ Satisfação com Relacionamento Familiar } & Sim & $0,28(0,03-2,0)$ \\
\hline & Não & \\
\hline \multirow[t]{2}{*}{ Número de Dependentes } & Nenhum & $1,0(0,35-2,86)$ \\
\hline & 1 ou mais & \\
\hline Número de Pessoas da Família & Nenhum & $0,44(0,15-1,28)$ \\
\hline Que Trabalham & 1 ou mais & \\
\hline \multirow[t]{2}{*}{ A Moradia é } & Própria & $1,16(0,16-9,95)$ \\
\hline & Alugada / ou de Parentes & \\
\hline \multirow[t]{2}{*}{ Exerceu Atividade Remunerada } & Sim & $0,64(0,19-2,16)$ \\
\hline & Não & \\
\hline \multirow[t]{2}{*}{ Recebendo Aposentadoria } & Sim & $0,59(0,20-1,74)$ \\
\hline & Não & \\
\hline \multirow[t]{2}{*}{ Recebendo Pensão } & Sim & $1,72(0,55-5,37)$ \\
\hline & Não & \\
\hline \multirow[t]{4}{*}{ Renda Domiciliar Mensal } & menos de 1 salário mínimo & $1,95(0,16-51,46)$ \\
\hline & resultado não significativo & \\
\hline & 1-3 salários mínimos & \\
\hline & 4-5 salários mínimos & \\
\hline
\end{tabular}

*Valor de referência / *Reference value. 
Tabela 4 - Razão de chances entre os fatores relativos à sociabilidade e lazer e a presença ou não de declínio cognitivo. Município de Viçosa - MG (2005).

Table 4 - Odds ratio among the factors relative to sociability and leisure and the presence or not of cognitive decline. Municipal district of Viçosa, Minas Gerais (2005).

\begin{tabular}{|c|c|c|c|}
\hline Variáveis & Categorias & (IC 95\%) & OR \\
\hline \multirow[t]{2}{*}{ Rádio } & Sim & $(0,46-4,63)$ & 1,46 \\
\hline & Não & & \\
\hline \multirow[t]{2}{*}{ TV } & Sim & $(0,58-5,46)$ & 1,76 \\
\hline & Não & & \\
\hline \multirow[t]{2}{*}{ Ler Jornal, Revista, Livro } & Sim & $(0,12-1,24)$ & 0,40 \\
\hline & Não & & \\
\hline \multirow[t]{2}{*}{ Visitar Parentes } & Sim & $(0,35-4,26)$ & 1,20 \\
\hline & Não & & \\
\hline \multirow[t]{2}{*}{ Andar Pelo Bairro } & Sim & $(0,27-3,01)$ & 0,92 \\
\hline & Não & & \\
\hline \multirow[t]{2}{*}{ Ir à lgreja } & Sim & $(0,27-4,15)$ & 1,04 \\
\hline & Não & & \\
\hline \multirow[t]{2}{*}{ Ir à Jogos } & Sim & $(0,13-2,28)$ & 0,57 \\
\hline & Não & & \\
\hline \multirow[t]{2}{*}{ Fazer Compras } & Sim & $(0,0-13,14)$ & 0,87 \\
\hline & Não & & \\
\hline \multirow[t]{2}{*}{ Passeios Longos } & Sim & $(0,29-3,79)$ & 1,06 \\
\hline & Não & & \\
\hline \multirow[t]{2}{*}{ Outras Atividades } & Sim & $(0,15-1,32)$ & 0,45 \\
\hline & Não & & \\
\hline \multirow[t]{2}{*}{ Satisfeito com as Atividades Que faz } & Sim & $(0,12-2,45)$ & 0,54 \\
\hline & Não & & \\
\hline \multirow[t]{5}{*}{ Motivo da Insatisfação Com as Atividades } & *Problema de Custo & $(0,18-4,48)$ & 1,0 \\
\hline & Problema de Saúde & $(0,08-10,34)$ & 0,89 \\
\hline & Falta de Motivação & $(0,23-16,42)$ & 0,89 \\
\hline & Problema com Transporte & & 1,78 \\
\hline & Outras Razões & & \\
\hline
\end{tabular}

*Valor de referência / * Reference value.

de corte sejam encaminhados para avaliação neuropsicológica mais detalhada ${ }^{20}$. No presente estudo, os idosos diagnosticados com declínio cognitivo foram encaminhados para essa avaliação.

Dos idosos entrevistados, a maioria tem baixa escolaridade. Este resultado confirma a situação verificada nos países em desenvolvimento, inclusive o Brasil, onde $41,1 \%$ da população acima de 60 anos era analfabeta ${ }^{27}$. Pereira et $\mathrm{al}^{28}$, em estudo sobre a qualidade de vida dos idosos no município de Teixeiras, MG, identificaram que $71,1 \%$ dos entrevistados tinham menos de quatro anos de estudo, devendo ser considerados como analfabetos funcionais

No presente estudo, o resultado do MEEM foi influenciado pela variável escolaridade, o que vem sendo amplamente discutido na literatura ${ }^{7,8,21,22,26}$, uma vez que esse viés dificulta a interpretação dos seus resultados em idosos com baixa escolaridade. Ao analisar a associação entre a escolaridade e o declínio cognitivo observou- 
se que uma maior escolaridade atua como fator de proteção.

Estudos vêm confirmando que, quanto mais jovem é o indivíduo e quanto maior seu nível de escolaridade, maior é o escore final do MEEM. Entretanto, alguns autores consideram que o baixo nível educacional e a idade são fatores de risco reais não só para o desempenho no MEEM mas para o desenvolvimento de demência $^{7,21,22,24,25}$.

Há uma tendência crescente, verificada na literatura, no sentido de se examinar a influência da escolaridade no desempenho do MEEM e, de um modo geral, tem-se concluído que um nível educacional elevado seria preventivo para o declínio cognitivo ${ }^{4,20}$.

Segundo Almeida ${ }^{7}$, a idade e a escolaridade se associam de forma significativa com o desempenho dos idosos no MEEM; entretanto, no presente estudo não se encontrou influência estatisticamente significante entre a variável idade e o declínio cognitivo. Uma possível hipótese explicativa pode se dever ao número de idosos na faixa etária entre 70-79 (32\%), sendo $3 \%$ de idosos com 80 anos ou mais, ou seja, uma pequena parcela se encontrava em idade mais avançada (Tabela 1).

Estudos com idosos confirmam uma maior participação do sexo feminino em relação ao masculino ${ }^{29}$. Lebrão e Laurenti ${ }^{24}$ ressaltam que, no Brasil, o número absoluto de mulheres idosas tem sido superior quando confrontado com o de homens acima de 65 anos. Isso pode acontecer pela existência da mortalidade diferencial entre os sexos, inclusive na população brasileira. No estudo sobre saúde, bem-estar e envelhecimento realizado em São Paulo com 2.143 idosos, foram encontrados $58,6 \%$ de mulheres, percentual bastante próximo ao encontrado no Censo de 2000, que foi de 59,5\%. As mulheres constituem a maior parte da população idosa mundial e vivem em média sete anos mais do que os homens; portanto, o envelhecimento passa a ser um fenômeno que se conjuga antes de tudo no feminino ${ }^{30}$.

Segundo o IBGE ${ }^{27}$, no censo de 2000, as mulheres de 60 anos e mais representavam $9,28 \%$ da população total e os homens 7,82\%. A predominância da população feminina entre idosos tem causado repercussões nas demandas por políticas públicas de saúde, pois as mulheres estão mais sujeitas a deficiências físicas (paraplegia e cegueira) e transtornos mentais (homens $8 \%$ e mulheres $12,39 \%$ ), há um maior número de mulheres morando sozinhas em decorrência da viuvez, elas têm menor nível de escolaridade, menor experiência de trabalho no mercado formal e menor acesso a aposentadoria, o que requer maior assistência tanto do Estado quanto das famílias $^{31}$.

Buscando conhecer a rede de suporte familiar dos idosos, verificou-se que, em relação à situação conjugal e ao arranjo familiar no domicílio, os resultados mostraram-se semelhantes aos de Santos et al. ${ }^{32}$, em estudo realizado sobre qualidade de vida na terceira idade. Os autores constataram que $55,5 \%$ dos idosos convivem com outros familiares em seu domicílio, sendo que $28 \%$ destes vivem com seus cônjuges e $15 \%$ vivem sozinhos.

Um dado positivo é que a maioria dos idosos entrevistados (86\%) se diz satisfeita com o seu relacionamento familiar. $\mathrm{Ob}$ serva-se que a maioria desses idosos tem dependentes e suas aposentadorias constituem importante fonte de renda, ou mesmo a principal renda da família. Pesquisas sobre o envelhecimento e a qualidade de vida mostram a valorização por parte dos idosos do bom relacionamento com a família ${ }^{33-35}$. Vecchia et al. ${ }^{35}$ realizaram um inquérito, em Botucatu, SP, sobre o significado da qualidade de vida para os idosos e identificaram que $49 \%$ dos entrevistados mencionaram que qualidade de vida está primeiramente associada aos relacionamentos interpessoais, ou seja, os idosos priorizam a questão afetiva e a família.

Para os idosos, ter casa própria é um fator de segurança e satisfação. No presente estudo, $82 \%$ dos entrevistados possuíam casa própria, o que significa o acúmulo de patrimônio pela maioria, embora sejam de 
baixa renda. Esses resultados foram superiores aos encontrados por Santos et al. ${ }^{32,36}$.

Os resultados das variáveis econômicas são condizentes com a situação enfrentada pela maioria dos idosos no Brasil, isto é, possuem baixo poder aquisitivo, sendo que as aposentadorias e pensões constituem sua principal fonte de renda. Segundo o IBGE $^{27}$, em 2003, 43,8\% dos idosos tinham rendimento inferior a um salário mínimo.

Davim et al. ${ }^{29}$ citam que a problemática dos idosos também está relacionada às aposentadorias precoces, de baixos valores, que constituem sua principal fonte de rendimentos, impossibilitando o atendimento de suas necessidades.

No que diz respeito à renda domiciliar mensal constatou-se um nível relativamente baixo (2,3 salários mínimos em média), sendo que $66 \%$ dos idosos têm pelo menos um ou mais dependentes, e 3, em média, residem no domicílio. Um nível de renda baixo em idosos também foi encontrado em outros estudos, como o de Laurenti e Lebrão ${ }^{24}$, que constatou que a renda dos idosos era de 2,1 salários mínimos, em média, sendo que grande parte deles era responsável pelo sustento familiar. Já Pereira et al. ${ }^{28}$ constataram que $61,1 \%$ dos idosos tinham renda mensal igual ou inferior a um salário mínimo.

Destaca-se a importância de se analisar a sociabilidade e o lazer, pois esses são indicadores da qualidade de vida e, portanto, influenciam o estado de saúde. Neste estudo verificou-se que a prática religiosa é um fator de grande influência nessa fase da vida, figurando entre as atividades mais mencionadas, juntamente com visitas a parentes e assistir televisão. No entanto, os problemas mais citados como principais empecilhos para uma maior satisfação com o cotidiano foram a falta de recursos e problemas de saúde. Na pesquisa de Santos et al. ${ }^{32}, 100 \%$ dos idosos demonstraram afinidade com algum tipo de atividade ou prática religiosa, predominando o engajamento na religião católica, com $87,5 \%$. A prática de uma religião por parte dos idosos permite-lhes estabelecer um relacionamento social fora do ambiente familiar, facilitando a interação com novas amizades e permitindo-lhes as trocas de experiências, o que favorece seu bem-estar e autonomia, ajudando-os a vencer com mais facilidade as dificuldades dessa etapa da vida.

Para Campos ${ }^{37}$, a compreensão de que o lazer é uma prática que estimula a criatividade e favorece os relacionamentos interpessoais, torna-o uma alternativa concreta para a melhoria da qualidade de vida, na medida que enseja motivação para que se continue ativo, produtivo e interessado, convergindo para a realização pessoal.

Dados epidemiológicos constatam que, para uma boa qualidade de vida, é imprescindível a auto-suficiência no cuidado consigo mesmo, destacando-se a importância da manutenção de diferentes opções de atividades de lazer como um fator de autonomia e bem-estar emocional. Além disso, sociabilidade, lazer e educação (como estratégia de socioterapia e de manutenção da "mente ativa") tornam-se dispositivos privilegiados de intervenção junto a esta população e ganham espaço significativo na mídia para a criação e divulgação de uma "nova velhice"38.

A saúde de um indivíduo resulta de condições biológicas, socioculturais e psíquicas $^{32}$. Nesse sentido, é possível, então, levantar a hipótese de que o convívio social e diferentes atividades de lazer podem contribuir como mais um fator de proteção para o declínio cognitivo, porque estes aspectos ajudam a manter o idoso autônomo dentro do seu contexto familiar e sociocultural, o que é fundamental para suas funções cognitivas e para seu bemestar psicológico. Sugere-se, então, que novos estudos sejam feitos para comprovar esta hipótese.

\section{Conclusão}

O perfil da população deste estudo é predominantemente do sexo feminino, com baixo nível de escolaridade, baixa ren- 
da e idade entre 60-69 anos. Observou-se que a maioria dos idosos tem companheiros e/ou convivem com outros familiares em seu domicílio e, ainda, relatam estar satisfeitos com o relacionamento familiar. Quanto à sociabilidade e ao lazer, verificou-se que a grande maioria considera-se satisfeita com sua vida social e lazer no cotidiano, apesar de se restringirem às práticas religiosas, a visitar parentes e assistir televisão.

Em vista dos resultados obtidos, podese inferir que um maior nível de escolaridade pode atuar como um fator de proteção para o declínio cognitivo. No entanto, o elevado número de idosos com declínio cognitivo detectado pelo MEEM, revela que este teste é influenciado pela variável escolaridade e, como está mostrado em outros estudos, o ponto de corte utilizado deve ser ajustado à escolaridade. Deve-se considerar que o MEEM é um instrumen- to de rastreio do comprometimento das funções cognitivas; portanto, para se concluir um diagnóstico de demência em idosos cujas respectivas pontuações foram abaixo do determinado, é necessária uma avaliação neuropsicológica mais apurada.

Contudo, há necessidade de um enfoque mais amplo dos fatores que proporcionam um envelhecimento saudável, pois este é resultado da interação multidimensional entre a saúde física e mental, autonomia, integração social, suporte familiar e independência econômica. Assim, sugerem-se novas investigações sobre o declínio cognitivo e seus impactos na qualidade de vida dos idosos. Além da importância para a orientação de políticas públicas de saúde, acredita-se que, conhecendo-se mais sobre essa enfermidade, mais se poderá trabalhar para a prevenção e para o diagnóstico precoce da demência.

\section{Referências}

1. Garrido R., Menezes P. O Brasil está envelhecendo: boas e más notícias por uma perspectiva epidemiológica. Rev Bras Psiquiatr 2002; 24(I): 3-6.

2. Organização das Nações Unidas. Informe da Assembléia Mundial sobre o envelhecimento da população. A - Conf. 31. Viena: ONU; 1982.

3. Brasil. Lei 8842, de 4 de janeiro de 1994. Disponível em: www.planalto.gov.br/CCIVIL/LEIS/L8842.htm (Acessado em 21 de setembro de 2006)

4. Cerqueira A, Oliveira N. Programa de apoio a cuidadores: uma ação terapêutica e preventiva na atenção à saúde dos idosos. Psicol USP 2002; 13(1): 1-11.

5. Argimon IIA, Stein LM. Habilidades cognitivas em indivíduos muito idosos: um estudo longitudinal. Cad Saúde Pública 2005; 21(1): 64-72.

6. Pelzer MT. A enfermagem e o idosos portador de demência tipo Alzheimer: desafios do cuidar no novo milênio. Est Interdiscipl Envelhec 2002; 4: 97-111.

7. Almeida OP. Mini exame do estado mental e o diagnóstico de demência no Brasil. Arq Neuro-Psiquiatr 1998; 56(3b): 605-12.

8. Nitrini R.. Epidemiologia da doença de Alzheimer no Brasil. Rev Psiquiatr Clín 1999; 26(5): 1-10.
9. Lopes MA, Botino CMC. Prevalência de Demência em diversas regiões do mundo. Análise dos estudos epidemiológicos de 1994 a 2000. Arq Neuro-Psiquiatr 2002; 60(1):61-9.

10. Macedo MBM, Ramos L.R.. Validade da versão em português da Clinical Dementia Rating. Rev Saúde Pública 2005; 39(6): 912-7.

11. Neto JG, Temelini MG, Forlenza OV. Diagnóstico diferencial das demências. Rev Psiquiatr Clín 2005; 32(3): $119-30$.

12. Dourado M et al. Consciência da doença na Demência. Resultados preliminares em pacientes com doença de Alzheimer leve e moderada. Arq Neuro-Psiquiatr 2005; 63 1): 114-8.

13. Veras $\mathrm{R}$ et al. Um envelhecimento digno para o cidadão do futuro. Rio de Janeiro: Relume- Dumará: UnATI/ Universidade Estadual do Rio de Janeiro; 1995.

14. Folstein MS, Folstein SE, McHugh PR. Mini-mental state: a practical method for grading the cognitive state of patients for the clinician. J Psychiatr Res1975; 12: 189-98.

15. Bertolucci PHF, Brucki SMD, Campacci SR, Juliano Y. O mini-exame do estado mental em uma população geral: impacto da escolaridade. Arq Neuro-Psiquiatr 1994; 52: 1 - 
16. Tombaugh TN, McIntyre NJ. The mini-mental examination: a comprehensive review. JAm Geriatr Soc 1992; 40: 922-35.

17. Instituto de Estudos e Orientação da Família (INEF). Demência. São Paulo: IDEF; 2002.

18. Charchat-Fichman H, Caramelli P, Sameshima K, Nitrini R. Declínio da capacidade cognitiva durante o envelhecimento. Rev Bras Psiquiatr 2005; 27(12): 79-82.

19. Abreu ID, Forlenza OV, Barros L.H. Demência de Alzheimer: correlação entre memória e autonomia. Rev Psiquiatr Clín 2005; 32(3): 131-6.

20. Brucki SMD, Nitrini R, Caramelli P, Bertolucci PHF, Okamoto IH. Sugestões para o uso do Mini- Exame do Estado Mental no Brasil. Arq Neuro-Psiquiatr 2003; 61(3B): 777-81.

21. Herrera JR E; Caramelli P, Nitrini R. Estudo epidemiológico populacional de demência na cidade de Catanduva, S.P. Rev Psiquiatr Clín 1998; 25(2): 70-3.

22. Laks J et al. O mini exame do estado mental em idosos de uma comunidade. Dados parciais de Santo Antônio de Pádua, Rio de Janeiro, RJ. Arq Neuro-Psiquiatr 2003; 61(3b): 782-5.

23. Lwanga SK, Lemeshow S. Sample size determination in health studies: a practical manual. Geneva: World Health Organization; 1982.

24. Laurenti R, Lebrão ML. Saúde, bem-estar e envelhecimento: o estudo SABE no município de São Paulo. Rev Bras de Epidemiol 2005; 8(2): 127-41.

25. Sotolongo PB, Carillo PC, Carillo CC. Deterioro cognitivo en la tercera edad. Rev Cubana Med Integr 2004; 20: 5-6.

26. Bustamante, SEZ, Bottino CMC, Lopes MA, Azevedo D, Hototian SR, Litvoc J et al. [FALTA TÍTULO DO ARTIGO] Arq Neuro-Psiquiatr 2003; 61(3A): 601-6.

27. Instituto Brasileiro de Geografia e Estatística (IBGE). Dados preliminares do censo de 2000. Disponível em: http://www.ibge.gov.br. (Acessado em Julho de 2006)
28. Pereira, RJ, Cotta RMM, Franceschini SCC, Ribeiro RCL, Sampaio RF, Priore SE, Cecon, PR. Contribuição dos domínios físico, social, psicológico e ambiental para a qualidade de vida global dos idosos. Rev Psiquiatr Rio Gd Sul 2006; 28(1): 27-37.

29. Davim RMB et al. Estudo com idosos de instituições asilares no município de Natal/RN: características socioeconômicas e de saúde. Rev Latino-Am Enfermagem 2004; 12(3): 518-24.

30. Ribeiro RCL et al.. Capacidade funcional e qualidade de vida de idosos. Est Interdiscipl Envelhec 2002; 4: 85-96.

31. Camarano AA, Yazaki LM. Envelhecimento da população brasileira: uma contribuição demográfica. Rio de Janeiro: IPEA; 2002.

32. Santos SR et al.. Qualidade de vida do idoso na comunidade: aplicação da Escala de Flanagan. Rev Latino-Am. Enfermagem 2002; 10(6): 754-64.

33. Caldas CP. Envelhecimento com dependência: responsabilidade e demandas da família. Cad Saúde Pública 2003; 19(3): 773-81.

34. Romero, DE. Diferenciais de gênero no impacto do arranjo familiar no status de saúde dos idosos brasileiros. Ciênc Saúde Coletiva 2002; 7(4): 777-94.

35. Vecchia RD et. al.. Qualidade de vida na terceira idade: um conceito subjetivo. Rev Bras Epidemiol 2005; 8(3): 246-52.

36. Telarolli Jr. R, Machado JCMS, Carvalho F. Perfil demográfico e condições sanitárias dos idosos em área urbana do Sudeste do Brasil. Rev Saúde Pública 1996; 30(5): 485-98.

37. Campos, TJ. Lazer e terceira idade: contributos do turismo no âmbito do programa Clube da Melhor Idade [dissertação de mestrado]. Campinas: Universidade Estadual de Campinas; 2003.

38. Barros RDB, Castro AD. Terceira idade: o discurso dos experts e a produção do "novo velho". Est Interdiscipl Envelhec 2002; 4: 113-24.

Recebido em: 14/11/06 Versão final reapresentada em: 17/07/07 Aprovado em: 10/09/07 